\title{
The design of an optimal insurance contract for irreplaceable commodities
}

\author{
Rachel J. Huang* • Larry Y. Tzeng
}

Received: 25 February 2004 / Revised: 15 February 2006

(C) Springer Science + Business Media, LLC 2006

\begin{abstract}
This paper discusses optimal insurance contract for irreplaceable commodities. To describe the dual impacts on individuals when a loss occurs to the insured irreplaceable commodities, we use a state-dependent and bivariate utility function, which includes both the monetary wealth and sentimental value as two arguments. We show that over (full, partial) insurance is optimal when a decrease in sentimental value will increase (not change, decrease, respectively) the marginal utility of monetary wealth. Moreover, a non-zero deductible exists even without administration costs. Furthermore, we demonstrate that a positive fixed reimbursement is optimal if (1) the premium is actuarially fair, (2) the monetary loss is a constant, and (3) the utility function is additively separable and the marginal utility of money is higher in the loss state than in the no-loss state. We also characterize comparative statics of fixed-reimbursement insurance under an additively separable preference assumption.
\end{abstract}

Keywords Deductible · Optimal insurance contract · Fixed-reimbursement insurance $\cdot$ Irreplaceable commodities

JEL Classification G22 · D86

\section{Introduction}

Cook and Graham [1977] defined an irreplaceable commodity as one that holds sentimental value for its owner in addition to market value. Therefore, the loss of an irreplaceable commodity will strike its owner from two directions: One is a decrease

*The author acknowledge funding from National Science Council in Taiwan (NSC93-2416-H-130-020).

R. J. Huang $(\square)$

Department of Finance, Ming Chuan University, Taiwan

e-mail: rachel@mcu.edu.tw

L. Y. Tzeng

Department of Finance, National Taiwan University, Taiwan 
in sentimental value; the other is a decrease in monetary wealth. For example, an antique is irreplaceable. In the case of the loss of a painting by Monet, the owner of the painting will suffer not only from psychological depression but also from a huge loss of monetary wealth. Another example is health. A dreaded disease could causes an individual to feel pain and suffering and could decrease his/her salary due to the poor or declining health. Thus, optimal insurance contract for irreplaceable commodities may differ from that for replaceable commodities.

Shioshansi [1982] and Schlesinger [1984] pioneered the study of optimal insurance contracts for irreplaceable commodities and provided many ingenious insights into this issue. However, both of their papers exogenously assume that the optimal form of insurance is coinsurance. In this paper, we investigate the optimal insurance contract for irreplaceable commodities by adopting an endogenous indemnity function. Endogenously determining the form of indemnity, Raviv [1979] showed that, with transaction costs, the optimal insurance contract between a risk-averse insurer and an insured involves a deductible and coinsurance above the deductible. He also showed that an insurance contract with an upper limit could not be optimal. In this paper, we intend to find the optimal insurance contract for irreplaceable commodities by using Raviv's [1979] approach.

Shioshansi [1982] and Schlesinger [1984] assumed that one can always find monetary compensation equivalent to the loss of sentimental value. Therefore, the individual's preference is affected by the total value of the irreplaceable commodities, which is the sum of the sentimental value and the market value. However, while the individual can receive monetary indemnity, the loss of the insured commodity may not be replaced in some cases, such as losing an arm on an accident. To capture the nature of irreplaceable commodities, we employ a bivariate utility for individuals; and the two arguments are the net monetary wealth and net sentimental value of the insured objects.

We find that the optimal insurance contract for irreplaceable commodities contains a deductible and coinsurance above the deductible. This finding demonstrates that Raviv's result [1979] is robust no matter if the insured object is irreplaceable. Moreover, we show that a non-zero deductible could still exist even without any administration costs. Our result provides a new rationale for the existence of the deductible. We further analyze the coinsurance rate in the optimal contract. We find that over (full, partial) insurance is optimal when a decrease in sentimental value will increase (not change, decrease, respectively) the marginal utility of monetary wealth.

In addition, an insurance contract that specifies a fixed reimbursement is very common for dreaded disease insurance, term life insurance, and jewelry insurance, where the insured objects share the properties of irreplaceable commodities. Thus, this paper will demonstrate the conditions that ensure a fixed-reimbursement insurance as optimal. Gollier [1996] provided another rationale for the existence of fixedreimbursement insurance. He studied the optimal insurance contract where the indemnity is based on only a signal of the loss but not on the loss itself. From Gollier's results, it can be shown that a fixed indemnity is optimal when the signal is independent of the amount of loss. Following Gollier's unobservable loss-amount setting, Eeckhoudt, Mahul, and Moran [2003] characterize the properties for fixed-reimbursement insurance. In this paper, we consider the case where the loss is observable rather than 
unobservable. ${ }^{1}$ We focus on whether the optimal insurance contract could take the form of fixed-reimbursement when the insured object is irreplaceable.

This paper proceeds as follows. We develop our model in Section 2 and construct the optimal insurance contract in Section 3. Section 4 discusses the case of fixedreimbursement insurance and provides comparative statics. Section 5 concludes this paper.

\section{Model}

Assume that there is one risk-averse insured and one risk-neutral insurer in the insurance market. The insured object is an irreplaceable commodity with sentimental value $S$ to its owner. Assume an observable loss $x$ occurs with probability $\pi$, and $0<\underline{L} \leq x \leq S$, where $\underline{L}$ denotes the minimum damage. At the loss state, not only does the sentimental value of the commodity to the individual decrease from $S$ to $S-x$, but the agent's monetary wealth also declines by $g(x), g(x) \geq 0$ and $g^{\prime}(x) \geq 0$.

The insurance contract specifies the premium $P$ and the indemnity that is monetary compensation to the insured if the loss occurs. Since $x$ is observable, the compensation scheme can be a function of $x$, denoted as $I(x), I(x) \geq 0$. Raviv [1979] assumed that there is an upper limit condition for $I(x)$ : The compensation scheme could not be greater than the monetary loss, i.e., $I(x) \leq g(x)$. However, we release this condition as in Schlesinger [1984] and allow that the insurer may pay more than the monetary loss. In the dreaded disease example, the insurer could agree upon a contract that compensates the individual's salary loss and the utility loss due to the decrease in sentimental value. Assume the administration cost is proportional to the coverage. Thus, the premium charged by a risk-neutral insurer would be written as $P=\pi(1+\lambda) \int_{L}^{S} I(x) f(x) d x$, where $\lambda \geq 0$ is a loading factor and $f(x)$ is the conditional probability density function of $x$ when a loss is obtained.

To reflect the nature of irreplaceable commodities, we adopt a bivariate utility function for the individual. The net monetary wealth and net sentimental value are the two arguments. We further assume that the insured's utility function in the no-loss state differs from that in the loss state. Thus, after purchasing insurance, the insured's utility function is $\mu(w-P, S)$ if no loss occurs and $u(w-P+I(x)-g(x), S-x)$ otherwise, where $w$ is the initial monetary wealth of the insured. Assume that $\mu_{i}>0$, $u_{i}>0, \mu_{i i}<0, u_{i i}<0, i=1,2$, where $\mu_{i}$ and $u_{i}$ represent the marginal utility of the $i$-th component; $\mu_{i i}$ and $u_{i i}$ represent the second derivative of the utility of the $i$-th component.

The optimal policy is obtained by maximizing the insured's expected utility ${ }^{2}$

$$
\max _{I(x)} E U=(1-\pi) \mu(w-P, S)+\pi \int_{\underline{L}}^{S} u(w-P+I(x)-g(x), S-x) f(x) d x
$$

\footnotetext{
${ }^{1}$ To clearly demonstrate the existence of fixed-reimbursement insurance, we also assume that there is no moral hazard problem in the model.

2 The participation condition for buying insurance, $E U \geq(1-\pi) \mu(w, S)+\pi \int_{\underline{L}}^{S} u(w, S-x) f(x) d x$, is assumed to hold.
} 


$$
\begin{aligned}
& \text { s.t. } \quad P=\pi(1+\lambda) \int_{\underline{L}}^{S} I(x) f(x) d x, \\
& \quad I(x) \geq 0 .
\end{aligned}
$$

\section{Optimal insurance}

From the model setting, we can obtain the first proposition.

Proposition 1. The optimal insurance contract $I^{*}(x)$ contains deductible D and partial coverage above the deductible. The marginal coverage above the deductible satisfies the following condition:

$$
I^{*^{\prime}}(x)=\frac{u_{12}}{u_{11}}+g^{\prime}(x), \text { for } D<x \leq S,
$$

where $u_{12}$ is the change of the marginal utility of good 1 when the quantity of good 2 changes.

Proof: The proof of the optimal contract is similar to the proof of Proposition 2, and, therefore, is omitted here. According to Raviv [1979], the proportional costs ensure a deductible. Thus, we demonstrate only the result for $I^{*^{\prime}}(x)$. The first-order condition for an inner solution of $I(x)$ is that $u_{1}\left(w-P+I^{*}(x)-g(x), S-x\right)$ is equal to a constant, $\forall x$. Differentiate this equation with respect to $x$ and obtain the optimal marginal indemnity function as described.

Let us first focus on the discussion of coinsurance above the deductible. Proposition 1 demonstrates that the indemnity above the deductible is not necessarily equal to the monetary loss. This depends on the sign of $u_{12}$.

When $u_{12}<0$, we have $I^{*^{\prime}}(x)>g^{\prime}(x)$, where the marginal indemnity is larger than the marginal monetary loss. Over insurance ${ }^{3}$ becomes optimal in this case. $u_{12}<0$ demonstrates that a decrease in sentimental value will increase the marginal utility of monetary wealth. Thus, the insured would demand more monetary compensation in the loss state, which results in overinsurance. In the case of $u_{12}<0$, the monetary wealth and the sentimental value could be viewed as "substitution goods" in economic intuition.

If $u_{12}=0$ (an additively separable utility illustrates the case), then $I^{*^{\prime}}(x)=g^{\prime}(x)$. Thus, the optimal indemnity $I^{*}(x)=\max [g(x)-g(D), 0]$. Since $u_{12}=0$ means a decrease in sentimental value will not affect the marginal utility of monetary wealth, obviously the monetary compensation scheme will consider only the monetary loss.

If $u_{12}>0$, then $I^{*^{\prime}}(x)<g^{\prime}(x)$. Partial insurance becomes optimal. When a decrease in sentimental value will decrease the marginal utility of monetary value, the individual would demand less compensation for an additional loss. Thus, optimally the marginal

\footnotetext{
${ }_{3}$ Through out this paper, we define over (respectively, full, partial) insurance by $I^{*^{\prime}}(x)>$ (respectively, $=,<) g^{\prime}(x)$. 
monetary compensation will be less than the marginal monetary loss. In this case, the sentimental value and monetary wealth are considered as "complementary goods" in the view of economics.

In conclusion, Proposition 1 shows that coverage above the deductible could be over, full, or partial insurance for irreplaceable commodities; and the results depend on the degree of substitutability or complementarity between the sentimental value and monetary wealth.

It is noticed that this model may allow a positive $I(\underline{L})$ in equilibrium. First, the model does not assume that $I(x) \leq x$. Second and moreover, the utility function in the no-loss state, $\mu$, may not be the same as that in the loss state, $u$. Thus, $I(\underline{L})$ could be positive no matter the deductible exists. In the case of zero deductible, a fixedreimbursement insurance contract, which contains a constant coverage for all level of losses, could be observed under $u_{12}=0$.

The non-zero deductible exists in Proposition 1 because of a non-constant administration cost, as predicted by the literature. The next Proposition will demonstrate that a non-zero deductible may still be observed even without any administration costs.

Proposition 2. When the premium is actuarially fair and $-u_{11}(w-P-g(x), S-$ $x) g^{\prime}(x)-u_{12}(w-P-g(x), S-x)>0, \forall x$, the optimal insurance contract still contains a deductible if and only if

$$
\int_{\underline{L}}^{S} u_{1}\left(w-P^{*}+I^{*}(x)-g(x), S-x\right) f(x) d x<\mu_{1}\left(w-P^{*}, S\right) .
$$

Proof: There are two steps in this proof. In the first step, we prove that the optimal insurance contract under a no-loading case may still contain a deductible. In the second step, we provide the condition for a positive deductible.

When the premium is actuarially fair, the optimal problem (1) becomes

$$
\begin{aligned}
\max _{I(x)} E U & =(1-\pi) \mu(w-P, S)+\pi \int_{\underline{L}}^{S} u(w-P+I(x)-g(x), S-x) f(x) d x \\
\text { s.t. } \quad P & =\pi \int_{\underline{L}}^{S} I(x) f(x) d x, \\
& I(x) \geq 0 .
\end{aligned}
$$

Thus, the Hamiltonian for this problem is

$$
H=[u(w-P+I(x)-g(x), S-x)-\theta I(x)] f(x),
$$

where $\theta$ is the Hamiltonian multiplier and is a constant. Because the Hamiltonian is concave in $I(x)$, the necessary and sufficient conditions for a maximum are

$$
\begin{gathered}
\mathbf{L}=u_{1}(w-P-g(x), S-x)-\theta \leq 0 \quad \forall x, \quad I^{*}(x)=0 ; \\
\mathbf{M}=u_{1}(w-P+I(x)-g(x), S-x)-\theta=0 \quad \forall x, \quad I^{*}(x)>0 .
\end{gathered}
$$


Since we assume $-u_{11} g^{\prime}(x)-u_{12} \geq 0, \forall x, \mathbf{L}$ is an increasing function of $x$. We could define a deductible $D$, which satisfies

$$
u_{1}(w-P-g(D), S-D)=\theta \text {. }
$$

Differentiating Eq. (7) with respect to $x$, we could find the optimal positive indemnity satisfies Eq. (2). Thus, the optimal indemnity function will be

$$
\begin{array}{rlr}
I^{*}(x) & =0, & \text { if } x \leq D, \\
& >0, \text { and } I^{*^{\prime}}(x)=\frac{u_{12}}{u_{11}}+g^{\prime}(x), & \text { otherwise. }
\end{array}
$$

This completes the first step. Now we would like to show the condition for a non-zero deductible. The optimal deductible is decided by maximizing the following objective function:

$$
\begin{aligned}
\max _{D} E U= & (1-\pi) \mu\left(w-P^{*}, S\right)+\pi \int_{\underline{L}}^{D} u\left(w-P^{*}-g(x), S-x\right) f(x) d x \\
& +\pi \int_{D}^{S} u\left(w-P^{*}+I^{*}(x)-g(x), S-x\right) f(x) d x,
\end{aligned}
$$

where $P^{*}=\pi \int_{D}^{S} I^{*}(x) f(x) d x$. The derivative of Eq. (11) at $D=\underline{L}$ is

$$
\begin{aligned}
\pi(1-\pi)\left(\left.\frac{\partial I^{*}(x)}{\partial D}\right|_{D=\underline{L}}\right) & {\left[-\mu_{1}\left(w-P^{*}, S\right)\right.} \\
& \left.+\int_{\underline{L}}^{S} u_{1}\left(w-P^{*}+I^{*}(x)-g(x), S-x\right) f(x) d x\right] .
\end{aligned}
$$

Since $\frac{\partial I^{*}(x)}{\partial D}$ is negative, Eq. (12) will be positive if and only if the last term is negative.

Proposition 2 states the necessary and sufficient conditions for a non-zero deductible. A non-zero deductible exists if and only if the marginal utility of monetary wealth at the no-loss state, $\mu_{1}\left(w-P^{*}, S\right)$, is greater than the expected marginal utility of monetary wealth at the loss state, $\int_{\underline{L}}^{S} u_{1}\left(w-P^{*}+I^{*}(x)-g(x), S-x\right) f(x) d x$. Since $u_{11}<0$, the insured could be better off if he/she purchases less coverage at the loss state, i.e., a positive deductible will be a Pareto improvement. Moreover, the last term in Eq. (12) could be rewritten as

$$
\int_{\underline{L}}^{S}\left[-\mu_{1}\left(w-P^{*}, S\right)+u_{1}\left(w-P^{*}+I^{*}(x)-g(x), S-x\right)\right] f(x) d x,
$$


since $\mu_{1}\left(w-P^{*}, S\right)$ does not vary with $x$. If $\mu_{1}\left(w-P^{*}, S\right)<u_{1}\left(w-P^{*}+I^{*}(x)-\right.$ $g(x), S-x), \forall x$, then a non-zero deductible still exists in optimal. However, this condition is not necessary.

The critical assumptions that make a deductible optimal are the state-dependent and the bivariate utility function. If $\mu=u$ and $u_{12}=0$, then

$$
I^{*}=\max [g(x)-g(D), 0]
$$

It can be shown that eq. (12), the first-order condition evaluated at $D=\underline{L}$, becomes

$$
\pi(1-\pi)\left[-g^{\prime}(\underline{L})\right]\left[-u_{1}\left(w-P^{*}, S\right)+u_{1}\left(w-P^{*}-g(\underline{L}), S-x\right)\right]=0,
$$

where $g(\underline{L})=0$. A deductible will not appear.

On the other hand, whether $I(\underline{L})>0$ depends on not only the state-dependent and the bivariate utility function but also $g(\underline{L})$. If $g(\underline{L}) \geq 0$, we could generally find that $I(\underline{L})>0$.

\section{Fixed-reimbursement insurance}

In this section, we will demonstrate the conditions that ensure a fixed-reimbursement insurance being optimal for irreplaceable commodities and provide comparative statics. The next proposition illustrates the conditions.

\section{Proposition 3. If}

(1) $P=\pi \int_{L}^{S} I(x) f(x) d x,^{4}$

(2) $g(x)=k$, where $k$ is a constant and $k \geq 0$,

(3) $u_{12}=0$ and $-\mu_{1}(w, S)+u_{1}(w-k, S-x)>0, \forall x$, then the optimal contract specifies a fixed reimbursement.

Proof: Since $u_{12}=0, I^{*^{\prime}}(x)=g^{\prime}(x)=0$ from Proposition 1 . Therefore, it is obvious that $I^{*}(x)$ is a constant. The question is whether the constant is zero. Assume the coverage is $Q$. The premium will be $\pi Q$ since $P=\pi \int_{\underline{L}}^{S} Q f(x) d x$. The insured's expected utility is

$$
(1-\pi) \mu(w-\pi Q, S)+\pi \int_{\underline{L}}^{S} u(w-\pi Q+Q-k, S-x) f(x) d x .
$$

\footnotetext{
${ }^{4}$ This assumption is made for the sake of simplicity to demonstrate the existence of fixed-reimbursement insurance. Our results can be extended for the case with a positive loading. If there is a positive loading, $\lambda$, for insurance premium, condition (3) in Proposition 3 should be changed to $u_{12}=0$ and $-(1-\pi)(1+$ $\lambda) \mu_{1}(w, S)+(1-(1+\lambda) \pi) u_{1}(w-k, S-x)>0, \forall x$.
} 
The derivative of Eq. (16) at $Q=0$ is

$$
-\mu_{1}(w, S)+u_{1}(w-k, S-x)>0, \quad \forall x
$$

Thus, the optimal $Q$ is not zero.

Proposition 3 indicates that if (1) the premium is actuarially fair, (2) the monetary loss is a constant, ${ }^{5}$ and (3) the marginal utility of money at the loss state is irrelevant to the sentimental level ${ }^{6}$ and the individual's utility of money at the loss state is higher than at the no-loss state when no insurance is purchased, then the optimal insurance contract would be a fixed indemnity.

It should be noted that positive fixed reimbursement insurance could still exist even if $k=0$. For example, let $\mu\left(z_{1}, z_{2}\right)=-e^{-z_{1}}-e^{-z_{2}}$ but $u\left(z_{1}, z_{2}\right)=-a e^{-z_{1}}-e^{-z_{2}}$, where $a>1$ and $z_{i}$ represents the $i$-th argument, $i=1,2$, then the optimal fixed indemnity is $k+\ln a$. Moreover, if $k=0$, the optimal fixed indemnity is $\ln a$, which is still positive.

It is important to note that a fixed indemnity could not be developed in Raviv's [1979] setting. According to Raviv's Theorem 1, the optimal marginal indemnity for replaceable insured commodities, $I_{R}^{*^{\prime}}$, in the absence of transaction costs is as follows:

$$
I_{R}^{*^{\prime}}=\frac{R_{u}}{R_{u}+R_{v}} .
$$

In Eq. (18), $I_{R}^{*^{\prime}}$ refers to the optimal marginal indemnity under Raviv's model; and $R_{u}$ is the absolute risk aversion index for the insured. It is obvious that, for any finite $R_{v}$, the optimal marginal indemnity becomes zero only if $R_{u}=0$. That is, the insured is risk-neutral, which violates the general assumption in the literature about the insured's risk attitude.

To further derive comparative statics, we assume $\mu_{1}\left(z_{1}, z_{2}\right)=\psi^{\prime}\left(z_{1}\right)^{7}$ and $u_{1}\left(z_{1}, z_{2}\right)=a \psi^{\prime}\left(z_{1}\right)$, where $a$ is a constant, $a>1$, and $\psi\left(z_{1}\right)$ is a strictly concave function of $z_{1}$. Under this setting, the first-order condition for the insured's optimization problem (16) is

$$
-\psi^{\prime}(w-\pi Q)+a \psi^{\prime}(w-\pi Q+Q-k)=0 .
$$

Eq. (19) could be satisfied if and only if $Q^{*}>k$.

Both Eeckhoudt, Mahul, and Moran [2003] and our model point to fixedreimbursement insurance. However, the main mechanism that ensures a fixed

\footnotetext{
${ }^{5}$ An antique may be a proper example for $g(x)$ to be a constant. Sometimes, even a small bit of damage to an antique may wipe out all of its market value.

${ }^{6}$ This means that the utility at the loss state is additively separable. The separability assumption does not accurately describe individuals' consumption decisions for replaceable goods using the U.S. aggregation data, as in Diewert and Wales [1995] and Lewbel [1996]. However, as discussed by Rey and Rochet [2004], there is no consensus in the literature about the sign of the interaction between health (irreplaceable commodity) and wealth.

${ }^{7}$ Since the point here is to address the case of $u_{12}=0$, the introduction of $\psi^{\prime}$ does not further loss generality. 
Table 1 Comparative statics analysis

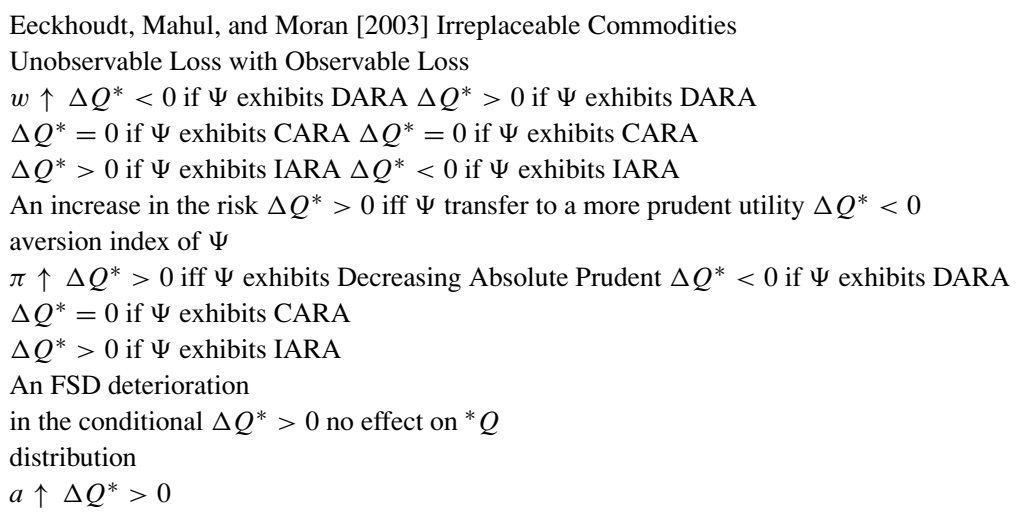

reimbursement is different. Eeckhoudt, Mahul, and Moran [2003] argue that the loss is unobservable, as assumed in Gollier [1996], whereas we assume it is observable. Moreover, we indicate that the fixed reimbursement appears because of the properties of an irreplaceable commodity to provide empirical hypotheses for further research. We further examine the comparative statics and compare them with Eeckhoudt, Mahul, and Moran [2003].

A summary of comparison is presented in Table 1. Table 1 demonstrates that with an additively separable utility function and under actuarially fair pricing, the insured will buy more insurance when he or she is richer if the utility associated with monetary wealth exhibits decreasing absolute risk aversion. At first glance, this contradicts the findings of Mossin [1968] and Eeckhoudt, Mahul, and Moran [2003]. Mossin [1968] showed that the insured purchases more (respectively, the same amount of, less) insurance with an increasing (respectively, constant, decreasing) absolute risk aversion. In fact, our finding is consistent with the finding in the literature.

In our model the insured has more monetary wealth in the loss state than in the no-loss state, i.e.,

$$
w-\pi Q^{*}+Q^{*}-k>w-\pi Q^{*}, \text { since } Q^{*}>k .
$$

On the contrary, under Mossin's [1968] and Eeckhoudt, Mahul, and Moran's [2003] setting, the insured has more monetary wealth in the no-loss state, i.e., initial wealth minus premium, than in the loss state, i.e., initial wealth minus premium plus coverage minus loss, where the coverage is no greater than the loss. If the utility associated with monetary wealth exhibits decreasing absolute risk aversion, the insured would allow for more of a wealth difference between the no-loss state and the loss state when he or she is richer. Thus, an increase in wealth induces an insured with a decreasing absolute risk-averse index to buy more insurance in our model, whereas it induces the insured to purchase less insurance in Mossin's [1968] and Eeckhoudt, Mahul, and Moran's [2003] model. In our model, fixed-reimbursement insurance is, indeed, a normal good if the insured's utility of money exhibits decreasing absolute risk aversion.

When the insured is more risk-averse, he or she would reduce the wealth difference between the loss and no-loss states in optima. Since our model ensures the insured's 
final wealth in the loss state is higher than in the no-loss state, the insured would purchase less insurance if he or she is more risk-averse. From Diamond and Stiglitz [1974], it can be shown that a risk-averse individual purchases proportionally more insurance. Eeckhoudt, Mahul and Moran [2003] also find that if $\psi$ becomes more prudent, then the optimal reimbursement will increase. In our model, a concave transform of the utility associated with money leads the insured to purchase less fixed-reimbursement insurance. Once again, our finding looks different from that in the literature because, in our model, the insured has more final wealth in the loss state than in the no-loss state.

An increase in $\pi$ contains a negative wealth effect only under the assumption of an actuarially fair premium. Table 1 shows that the insured should buy less insurance when the probability of risk occurrence decreases if the insured's utility of money exhibits decreasing absolute risk aversion. Since the effect of an increase in the probability of loss occurrence is in the opposite direction to that of an increase in initial wealth, an increase in $\pi$ increases the premium and results in a reduction in wealth.

Since the conditional distribution $f(x)$ plays an irrelevant role in determining the optimal fixed indemnity in our model, an FSD shift in the conditional distribution will not affect $Q^{*}$. However, it has a positive effect on $Q^{*}$ in Eeckhoudt, Mahul and Moran [2003]. An increase in $a$ implies that money is more important in the loss state and creates a higher utility for the insured. Thus, the insured would purchase more insurance.

\section{Conclusion}

In this paper we find the optimal insurance contract for irreplaceable commodities by adopting an endogenous indemnity function and a bivariate utility function with sentimental value and monetary wealth as two arguments. We show that the optimal marginal indemnity could be larger, equal to, or less than the marginal monetary loss. The results depend on the degree of substitutability or complementarity between the sentimental value and monetary wealth. When sentimental value and monetary wealth are viewed as substitution (complementary) goods, which are defined as $u_{12}<0$ $\left(u_{12}>0\right)$, the individual would be better off when he/she purchase over (less) insurance. Complementing the literature, we find that a deductible may still exist, even without any transaction costs, if and only if the marginal utility of monetary wealth at the no-loss state is greater than the expected marginal utility of monetary wealth at the loss state. Moreover, we demonstrate that if (1) the premium is actuarially fair, (2) the monetary loss is a constant, and (3) the utility function is additively separable and the marginal utility of money is higher in the loss state than in the no-loss state, then a positive fixed reimbursement is optimal under actuarially fair pricing. We further provide comparative statics of fixed-reimbursement insurance for irreplaceable commodities. Some of the comparative statics differ from the findings in the previous literature and would provide testable hypotheses for further empirical studies.

\section{References}

COOK, P.J. and GRAHM, D.A. [1977]: "The Demand for Insurance and Protection: The Case of Irreplaceable Commodities," Quarterly Journal of Economics, 91, 143-156.

DIAMOND, P.A. and STIGLITZ, J.E. [1974]: “Increases in Risk and in Risk Aversion,” Journal of Economic Theory, 8, 337-360.

글 Springer 
DIEWERT, W.E. and WALES, T.J. [1995]: "Flexible Functional Forms and Tests of Homogeneous Separability," Journal of Econometrics, 67, 259-302.

EECKHOUDT, L., MAHUL, O., and MORAN, J. [2003]: "Fixed-Reimbursement Insurance: Basic Properties and Comparative Statics," Journal of Risk and Insurance, 70, 207-218.

GOLLIER, C. [1996]: “Optimum Insurance of Approximate Losses,” Journal of Risk and Insurance, 63, 369-380.

LEWBEL, A. [1996]: “Aggregation Without Separability: A Standardized Composite Commodity Theorem," American Economic Review, 86, 524-543.

MOSSIN, J. [1968]: “Aspects of Rational Insurance Purchasing,” Journal of Political Economics, 4, 553568.

RAVIV, A. [1979]: “The Design of an Optimal Insurance Policy,” American Economic Review, 69, 84-96.

REY, B. and ROCHET, J. [2004]: "Health and Wealth: How do They Affect Individual Preferences"? The Geneva Papers on Risk and Insurance Theory, 29, 43-54.

SCHLESINGER, H. [1984]: “Optimal Insurance for Irreplaceable Commodities,” Journal of Risk and Insurance, 51, 131-137.

SHIOSHANSI, F.P. [1982]: "Insurance for Irreplaceable Commodities,” Journal of Risk and Insurance, 49, 309-320. 